\title{
An intermittency model for predicting roughness induced transition
}

\author{
Xuan Ge ${ }^{\mathrm{a}, *}$, Paul A. Durbin ${ }^{\mathrm{a}}$ \\ ${ }^{a}$ Aerospace Engineering, Iowa State University \\ Ames, Iowa 50011, USA
}

\begin{abstract}
An intermittency transport equation for RANS modeling, formulated in local variables, is extended for roughness-induced transition. To predict roughness effects in the fully turbulent boundary layer, published boundary conditions for $k$ and $\omega$ are used. They depend on the equivalent sand-grain roughness height, and account for the effective displacement of wall distance origin. Similarly in our approach, wall distance in the transition model for smooth surfaces is modified by an effective origin, which depends on equivalent sand-grain roughness. Flat plate test cases are computed to show that the proposed model is able to predict transition onset in agreement with a data correlation of transition location versus roughness height, Reynolds number, and inlet turbulence intensity. Experimental data for turbine cascades are compared to the predicted results to validate the proposed model.

Keywords: Laminar to turbulent transition, Intermittency, Wall roughness, Equivalent sand grain, Effective origin
\end{abstract}

\section{Introduction}

\subsection{The need for predicting rough wall, transitional boundary layers}

It is well known that surface roughness can trip a boundary layer. Nevertheless, there are few data correlations or prediction methods for roughness induced transition. They are needed for many applications. For instance, to increase the efficiency of turbomachinery performance, designers must account for effects of surface roughness on both heat transfer and aerodynamic loss. Experimental data show that roughness on in-service turbine blades can cause a considerable increase in heat load. Arts et al. (1990) point out that, at moderate Reynolds numbers, a smooth vane can have transition occurring far downstream of the leading edge on the suction side, even with high inflow turbulence intensities; but, as the roughness height increases, the onset of transition gradually moves upstream, to the leading edge. When the boundary layer becomes turbulent, heat transfer can increase by a factor of 10 (Stripf et al., 2009a).

\footnotetext{
*Corresponding author

Email addresses: xge@iastate.edu (Xuan Ge), durbin@iastate.edu (Paul A. Durbin)
} 
Boyle and Stripf (2009) mention that surface roughness generally decreases aerodynamic efficiency of a turbine blade cascade. But Boyle and Senyitko (2003) show that at low Reynolds numbers roughness improves aerodynamic efficiency, while at high Reynolds numbers roughness doubles vane loss. Therefore, to improve the efficiency at both low and high Reynolds numbers, it is necessary to properly represent the effects of roughness on the boundary layer.

\subsection{Approaches to calculate a fully turbulent boundary layer on a rough wall}

The model represents transition from laminar flow to turbulent flow over a rough wall. This requires a turbulence model that is applicable to a rough surface. In the present approach, the rough surface is replaced by an effective, smooth surface, on which new boundary conditions are imposed. They are a function of roughness height. One common approach to parameterize roughness is the equivalent sand grain roughness, which is adopted here.

Based on the sand grain roughness, Durbin et al. (2001) proposed a rough wall modification for the two layer $k-\epsilon$ model. An effective displacement of the wall distance origin was introduced and related to the sand grain roughness height through a calibration procedure. The effective displacement was related to a hydrodynamic roughness length, that is used to modify turbulence length scales and the boundary condition for $\epsilon$. The following equation was used to blend between the smooth and fully rough boundary conditions for $k$ :

$$
k_{w}=\frac{u_{*}^{2}}{\sqrt{C_{\mu}}} \min \left[1,\left(r^{+} / 90\right)^{2}\right],
$$

where $u_{*}$, defined as $u_{*}^{2}=\left.\left(\nu+\nu_{T}\right) \partial_{n} U\right|_{w}$ for rough walls, is the friction velocity, and $C_{\mu}=0.09 . r^{+}$is the dimensionless sand roughness height, $r u_{*} / \nu$, where $r$ is the dimensional roughness height.

Similar roughness boundary conditions for fully turbulent boundary layer have been proposed to extend the standard $k-\omega$ model: an early example is the Wilcox roughness modification (Wilcox, 1998); more recent models by Seo (2004) and Knopp et al. (2009) invoke the displacement of origin approach. While the Wilcox model requires a very fine mesh resolution and is not accurate for transitionally rough walls, the newer models give satisfactory results with near wall grid spacing similar to that for smooth walls.

Under fully rough conditions, the log-layer solution $k=u_{*}^{2} / \sqrt{C_{\mu}}$ extends to the effective wall origin, where the log-layer eddy viscosity $\nu_{T}=u_{*} \kappa\left(y+d_{0}\right)$ reduces to $u_{*} \kappa d_{0}$. Here $d_{0}$ is the effective displacement of the wall origin. $d_{0}$ can be determined analytically under fully rough conditions based on the shift of the velocity profile in the log-layer. This shift has been measured experimentally and fitted such that the new velocity profile can be written

$$
U / u_{*}=1 / \kappa \log (y / r)+8.5
$$

where $\kappa=0.41$. Then, if $d_{0}$ is defined in terms of $U$ by

$$
U / u_{*}=1 / \kappa \log \left(\left(y+d_{0}\right) / d_{0}\right)
$$


the last two equations give

$$
\frac{y+d_{0}}{d_{0}}=\frac{y}{r} e^{8.5 \kappa}
$$

Using the approximation $d_{0} \ll y$,

$$
d_{0}=e^{-8.5 \kappa} r \approx 0.03 r .
$$

From the definition $\nu_{T}=k / \omega$, the boundary condition for $\omega$ under fully rough condition should be

$$
\omega=\frac{u_{*}}{\sqrt{C_{\mu}} \kappa d_{0}} .
$$

Generally, the $\omega$ boundary condition represented as

$$
\omega_{w}=\frac{60 \nu}{\beta y_{\text {eff }}^{2}},
$$

where $y_{\text {eff }}=\max \left[y_{1}, y_{r}\right]$ in Knopp's model and $y_{\text {eff }}=\left(y_{1}+y_{r}\right)$ in Seo's. Here $y_{1}$ is the grid point next to the wall and

$$
y_{r}=\frac{\nu}{u_{*}}\left(\frac{60 \kappa \sqrt{C_{\mu}}}{\beta} d_{0}^{+}\right)^{1 / 2},
$$

to agree with (1.3), where $\beta=0.075$.

The variable $d_{0}^{+}$is a function of $r_{+}$that is obtained by calibration against the log-layer displacement, $\Delta U\left(r^{+}\right)$(Durbin, 2009). Knopp proposes

$$
d_{0}^{+}=0.03 r^{+} \min \left[1,\left(\frac{r^{+}}{30}\right)^{2 / 3}\right] \min \left[1,\left(\frac{r^{+}}{45}\right)^{1 / 4}\right] \min \left[1,\left(\frac{r^{+}}{60}\right)^{1 / 4}\right]
$$

Seo gives

$$
d_{0}^{+}=\left\{\begin{array}{cl}
0.56\left(\frac{r^{+}}{20}\right)^{2.5} & ; \quad 0 \leq r^{+}<20 \\
0.63 \zeta\left(r^{+}\right)+0.028 r^{+} & ; \quad 20 \leq r^{+}<90 \\
0.031 r^{+}-0.27 & ; \quad 90 \leq r^{+}
\end{array}\right.
$$

where $\zeta\left(r^{+}\right)=\sin \left[\pi\left(\left(r^{+}-20\right) / 70\right)^{0.9}\right]$. It is easy to see that $\omega_{w}$ decreases with increasing $r^{+}$, which leads to increase of $C_{f}$.

Under transitionally rough conditions, Knopp et al. (2009) use a linear blending function

$$
k_{w}=\frac{u_{*}^{2}}{\sqrt{C_{\mu}}} \min \left(1, r^{+} / 90\right)
$$

for the $k$ boundary condition, while Seo (2004) retains (1.1). 


\subsection{Modeling for roughness effects on transition}

A few recent studies propose roughness transition models. They are based on a data correlation for the momentum thickness Reynolds number at which transition starts. Its form is $R e_{\theta t-r o u g h}$ as a function of $R e_{\theta t-s m o o t h}$, surface roughness, and turbulence intensity. The correlation in Stripf et al. (2009a) depends on both the roughness height and density, while Boyle and Stripf (2009) propose a simpler formula, which only depends on the roughness height. The dimensionless roughness height used by the former is $r / \delta_{*}$, rather than the more general, $r_{+}$, used in equations (1.1), (1.6) and (1.5). Hence, in the next section, the model will be calibrated with the correlation proposed by Boyle and Stripf (2009),

$$
R e_{\theta t-\text { rough }}=\frac{R e_{\theta t-\text { smooth }}}{1+T u^{-0.625}\left(0.05\left(r^{+}-5\right)\right)^{1.25}},
$$

Here $R e_{\theta t-s m o o t h}$ is the critical $R e_{\theta t}$ for smooth walls proposed by Mayle (1991),

$$
R e_{\theta t-s m o o t h}=400 T u^{-0.625} .
$$

$T u$ is the free-stream turbulence intensity at the transition onset location, and $r^{+}$is the dimensionless roughness height defined in section 1.2. The term $r^{+}-5$ implies that a surface roughness can be considered hydraulically smooth if $r^{+}$is less than 5 . Also note that, by this correlation, transition onset becomes independent of the local turbulence intensity at high $r^{+}$values.

Herein, we extend the smooth wall, bypass transition model of Ge et al. (2014) and Durbin (2012) to account for the effect of wall roughness. Bypass transition skips the stage of Tollmien-Schilichting instability and is triggered by free-stream disturbances penetrating into the boundary layer and/or by surface roughness. The model of Ge et al. (2014) is based on the $k-\omega$ turbulence model and an intermittency transport equation. It uses only local variables and is tensorally invariant.

Inspired by the idea of the equivalent sand grain roughness and the displacement of origin approach for the roughness modification in fully turbulent flow, a displacement of origin method is developed for the intermittency equation. (However, the sink term in the intermittency equation needs a non-displacement type of modification.) Equations (1.4), (1.5) and (1.6) are chosen as the boundary conditions for $k$ and $\omega$ on rough walls.

Previously, Dassler et al. (2010) proposed a very different type of extension of a smooth wall transition model, which is known as the $\gamma-R e_{\theta t}$ model (Langtry and Menter, 2009; Menter et al., 2006). A transport equation was added on for a 'roughness amplification', $A_{r}$, that serves as a transition onset criterion. The production term of the transport equation for $\widetilde{R e_{\theta t}}$ in the $\gamma-R e_{\theta t}$ model was modified by a function of $A_{r}$.

A more recent paper Elsner and Warzecha (2014) introduced the roughness transition correlation by Stripf et al. (2009a) into the $\gamma-R e_{\theta t}$ model. However, the integral quantity $\delta_{*}$ has to be calculated at each time step, and provided at each point of the grid, so this model is not based on local variables. Both Dassler et al. (2010) and Elsner and Warzecha (2014) used the $k-\omega$-SST model, and they chose Wilcox's roughness boundary condition for the fully turbulent boundary layer. 


\section{Formulation of the model}

In this section, the details of the roughness modification will be presented and the rational will be provided. The modification consists of two steps: the first step is to add an effective displacement the origin, depending on the equivalent sand grain roughness height, to the wall distance. The data correlation (1.7) is used to calibrate the effective displacement. The second step is to modify some parameters in the sink term of the intermittency equation in order to make the model suitable to turbine blade cases where, there is a strong acceleration downstream of the stagnation point.

\subsection{Modification by the effective origin}

To modify the smooth wall transition model of Ge et al. (2014), $C_{r} r$ is added to the original model wherever the wall distance appears.

$$
\begin{gathered}
R_{\nu} \equiv \frac{\left(d+C_{r} r\right)^{2}|\Omega|}{2.188 \nu}, \\
R_{s} \equiv\left(d+C_{r} r\right) \cdot \frac{\mathbf{n}_{w} \cdot \nabla|S| \omega}{\sqrt{2}|S|^{2}},
\end{gathered}
$$

where $d$ is the true wall distance, $r$ is the equivalent sand grain roughness height, and $C_{r}$ is a constant coefficient. (See original definitions of $R_{\nu}$ and $R_{s}$ in the full model formulation, in the appendix.)

The key modification is to $R_{\nu} . R_{\nu}$ is a replacement for $R_{\theta}$, which is used to indicate transition onset in data correlations. $R_{\nu}$ is therefore a typical parameter for transition modeling based on only local variable. For smooth surfaces, near the wall, $R_{\nu} \rightarrow d_{+}^{2} / 2.188$ as $d_{+} \rightarrow 0$. For rough surfaces, $R_{\nu}$ invokes a displaced origin and becomes non-zero at the wall.

To calibrate the coefficient $C_{r}$ in equations (2.1) and (2.2), a flat plate test case with zero pressure gradient is adapted from the T3A setup in Ge et al. (2014). The original free-stream $T u$ for $\mathrm{T} 3 \mathrm{~A}$ is $3.5 \%$, but the transition location is more sensitive to roughness with lower free-stream $T u$. Therefore $T u=1.5 \%$ was selected for the present calibration. The inflow velocity $U_{i n}=5.2 \mathrm{~m} / \mathrm{s}$ and turbulence Reynolds number $R_{t}=\nu_{T} / \nu=14$ are kept unchanged. The predicted critical Reynolds number $R e_{\theta t}$ and the correlated $R e_{\theta t-r o u g h}$ from equation (1.7), are in good agreement when $C_{r}=0.26$. When evaluating $R e_{\theta t-r o u g h}$ using equation (1.7), $R e_{\theta t-s m o o t h}$ was not obtained from equation (1.8); instead, the predicted velocity profiles from simulations of smooth wall cases were integrated to compute $R e_{\theta t-s m o o t h}$ and thereafter to calculate $R e_{\theta t-\text { rough }}$.

Figure 2.1 shows how the coefficient $C_{r}$ determines the transition location: with small $C_{r}$, the critical Reynolds number $R e_{\theta t}$ is overestimated, whereas early transition is predicted with large $C_{r}$. This is understandable since $C_{r} r$ defines the effective wall displacement, which is an indicator of the extent to which surface roughness will affect the near wall region of the boundary layer. For given $r$, the larger $C_{r}$, the farther the wall origin extends into the flow, and the earlier the transition occurs. 


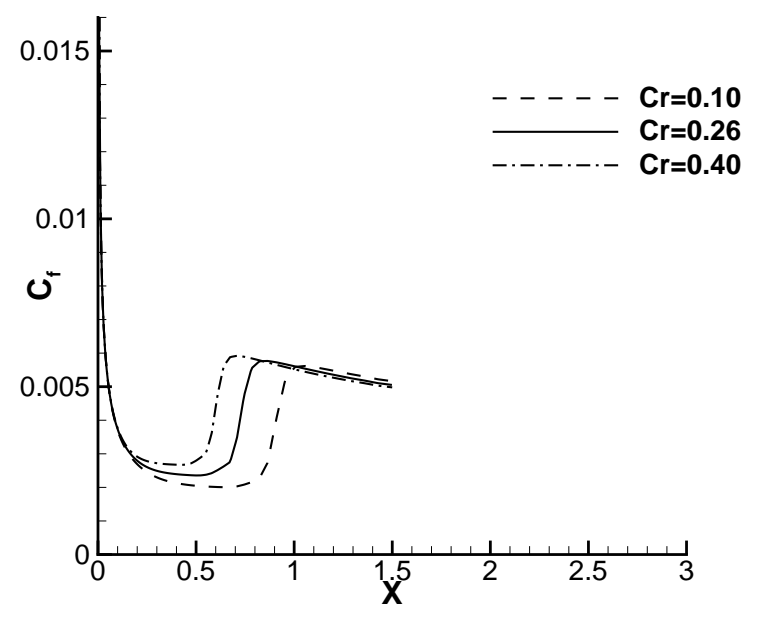

Figure 2.1: Effects of $C_{r}$. This test case is a flat plate case with inflow turbulence intensity $T u=1.5 \%$. The roughness height $r=10 \times 10^{-4} \mathrm{~m}$ is set unchanged for different $C_{r} \mathrm{~s}$.

However, this simple change is not enough, even for flat plate cases. As one may expect, a change may bring improvement in one place, but it may cause some issues in another. In this case, the sink term will not vanish after transition is complete, which leads to the intermittency function $\gamma$ not being unity. As a result, the skin friction will be underestimated in the fully turbulent region. The lower $R_{\nu}$ bound, in the function $G_{\gamma}$ of the appendix, is modified to fix this issue.

To shed light on how this modification works for the intermittency equation, the expression of the sink term of the intermittency equation (appendix) is recalled:

$$
E_{\gamma}=G_{\gamma} F_{\text {turb }}|\Omega| \gamma^{1.5}
$$

with $G_{\gamma}$ and $F_{\text {turb }}$ defined as

$$
\begin{gathered}
G_{\gamma-\text { smooth }}=7.5 \max \left[0, \min \left(100-R_{\nu}, 1\right)\right] \times \min \left[\max \left(R_{\nu}-18,0\right), 1\right], \\
F_{\text {turb-smooth }}=e^{-\left(R_{\nu} R_{t}\right)^{1.2}} .
\end{gathered}
$$

The subscript 'smooth' means the definition for a smooth wall, which will be changed for a rough wall. As mentioned above, when $r_{+}>5$, surface roughness will influence transition onset. Therefore, the sink term should be affected by the surface roughness. $G_{\gamma}$ switches from 0 to 7.5 in the range $18<R_{\nu}<100$. Near the wall this implies $18<d_{+}^{2} / 2.188<100$ or $6.3<d_{+}<14.8$. This needs to be adjusted.

To adjust the active region, the sink term is modified as

$$
\begin{array}{r}
G_{\gamma-t e m p 1}=7.5 \max \left[0, \min \left(100-R_{\nu}, 1\right)\right] \\
\times \min \left[\max \left(R_{\nu}-\left(18+R_{c 2}\right), 0\right), 1\right],
\end{array}
$$




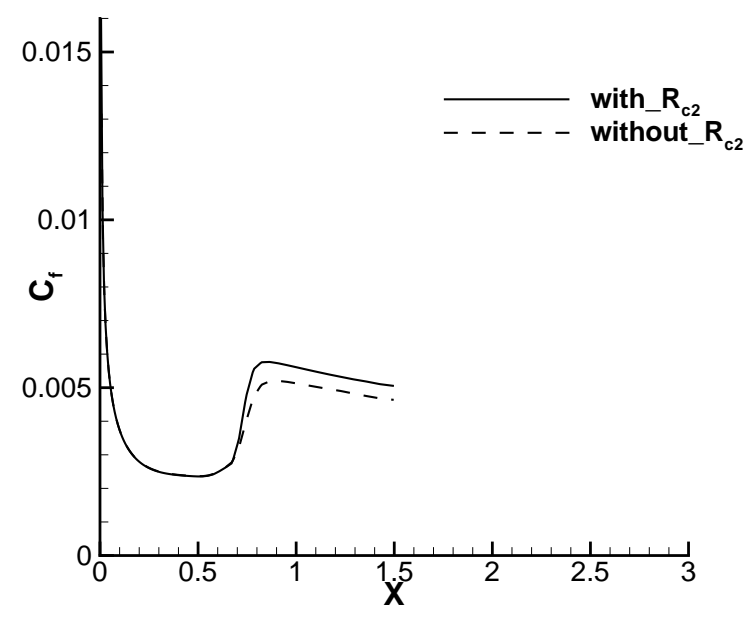

Figure 2.2: The effect of $R_{c 2}$. This test case is the same as figure 2.1.

$$
R_{c 2}=3.0\left[\frac{\left(C_{r} r\right)^{2}|\Omega|}{2.188 \nu}\right]^{0.8} .
$$

This switches off $G_{\gamma}$ in the near wall region after transition is complete, thereby making the sink term vanish appropriately. For smooth walls, $R_{c 2}$ becomes zero, eliminating the roughness effect. The contribution of $R_{c 2}$ is illustrated by figure 2.2. Without $R_{c 2}$, the $C_{f}$ curve is underestimated due to the existence of sink term in the fully turbulent region. (The $C_{f}$ curves in figure 2.1 were obtained with $R_{c 2}$ included in the sink term.)

The two coefficients - the multiplicative constant and the exponent of $R_{c 2}$-affect the results, as shown in figure 2.3. Figure 2.3(a) shows that the results are far less sensitive to the coefficient in $R_{c 2}$ than to the key coefficient $C_{r}$. As the selected value, 3.0, is halved or doubled, very slight differences are obtained. 4 times the selected value leads to early transition, because the lower bound of $R_{v}$ for $G_{\gamma}$ is too high and hence the sink term shrinks. 0.25 times the selected value will not shift the transition location, but this provides too small $R_{c 2}$ so that $C_{f}$ is underestimated in the fully turbulence region. Similarly, a larger value of the exponent causes earlier transition, and a lower value makes $C_{f}$ smaller after transition, as depicted in figure 2.3(b).

Note that the upper bound on $R_{\nu}$ in the function $G_{\gamma}$ has not been adjusted so far, because in the fully turbulent region $R_{\nu}=100$ generally occurs far away from the wall, where the sink term is driven to be zero by the factor $F_{\text {turb }}$. But it has to be modified for cases having a strong acceleration, like the region right after the leading edge of a turbine blade. This is explained in the next section. 


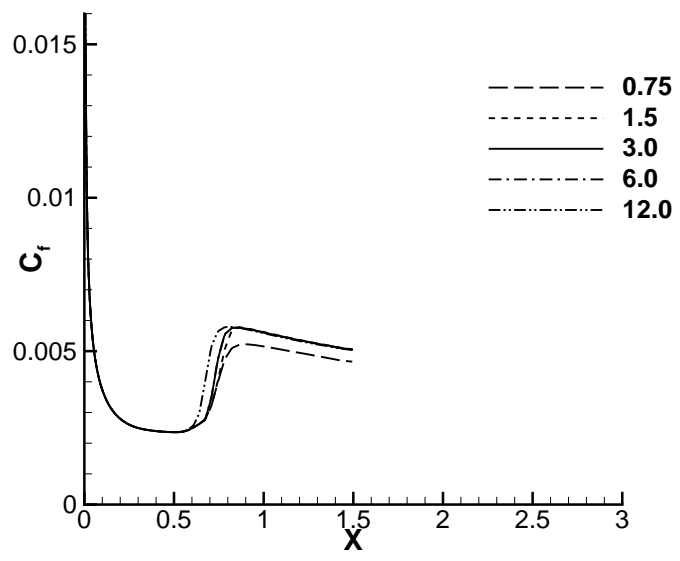

(a) Different values of the front coefficient of $R_{c 2}$.

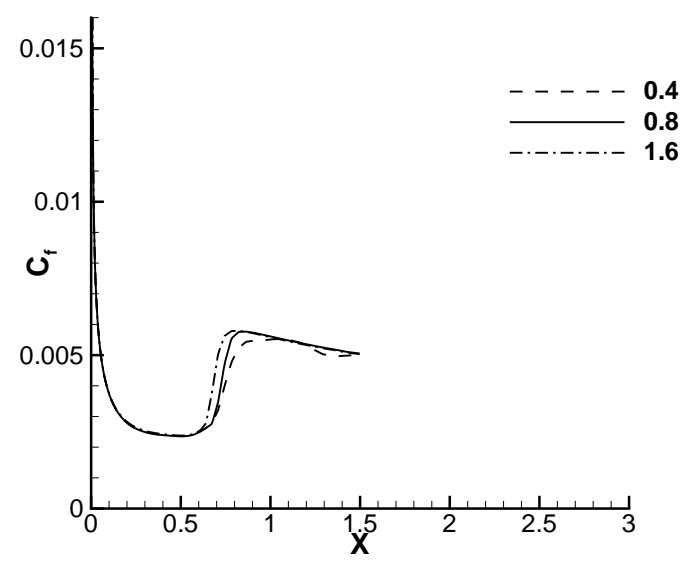

(b) Different values of the power coefficient of $R_{c 2}$.

Figure 2.3: The effects of coefficients in $R_{c 2}$. This test case is the same as figure 2.1.

\subsection{Modification for strong acceleration flows}

In some realistic cases, for example near the leading edge of a turbine blade, flow acceleration can be very large. A strong acceleration causes very large values of $|\Omega|$ in the near wall region (effectively, $r^{+}$becomes large). Consequently, $R_{\nu}$, defined by equation (2.1), becomes extremely large close to the wall if $r \neq 0$. Then the sink term will vanish right after the stagnation point, because both the factors, $F_{\text {turb }}$ and $G_{\gamma}$, of the sink term vanish for large $R_{\nu}$.

This can be overcome by decreasing $R_{\nu}$ in $F_{\text {turb }}$ and increasing the upper bound of $R_{\nu}$ in $G_{\gamma}$. It turns out that the former is more effective. We introduce a revision to $R_{\nu}$ so that $F_{\text {turb }}$ will not suppress the sink term in highly strained flow:

$$
\begin{gathered}
F_{\text {turb }}=e^{-\left(R_{\nu_{\text {new }}} R_{t}\right)^{1.2}}, \\
R_{\nu_{\text {new }}}=R_{\nu} e^{-F_{Q}^{1.5} / 350}, \\
F_{Q}=\max \left[0, \frac{r^{2} \sqrt{|Q|} \operatorname{sign}(Q)}{\nu}\right],
\end{gathered}
$$

where $Q=\Omega_{i j} \Omega_{i j}-S_{i j} S_{i j}$. $Q$ is the difference between the magnitude of the rate of rotation and the rate of strain. For zero pressure gradient flow, $Q$ is zero. For favorable pressure gradient flow, $Q$ is positive, and it is negative in adverse pressure gradients. Based on this property, $Q$ serves to indicate the high acceleration region on a turbine blade. Hence $F_{Q}$ is non-zero in a favorable pressure gradient and it decreases $R_{\nu_{\text {new }}}$. 


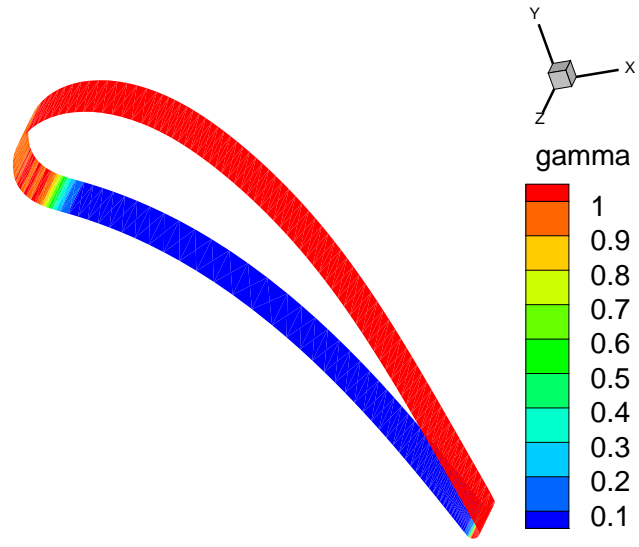

(a) Contours of $\gamma$ with old $F_{\text {turb }}$.

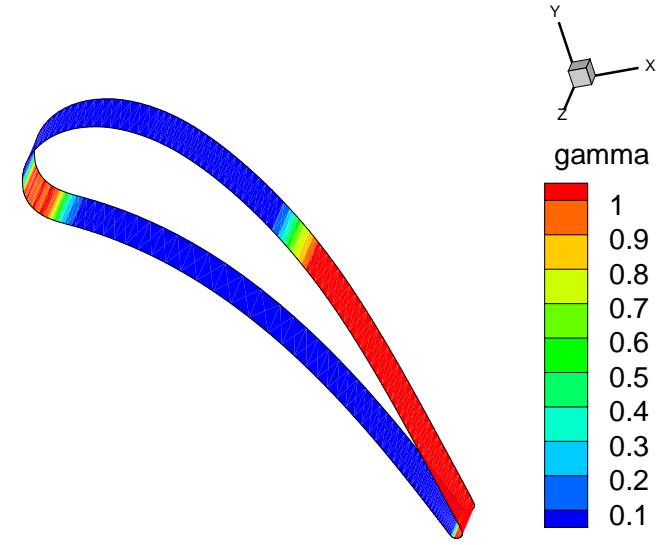

(b) Contours of $\gamma$ with new $F_{\text {turb }}$.

Figure 2.4: The effect of the modified $F_{\text {turb }}$. This test case uses the Stripf's turbine, with inflow chord Reynolds number $R e_{c}=1.4 \times 10^{5}$, turbulence intensity at the mid-pitch of the leading edge location $T u_{l . e .}=2.7 \%$, and roughness height $r=1.47 \times 10^{-4} \mathrm{~m}$.

Without this modification, the sink term would always vanish right after the stagnation point over the suction surface, even for low inflow Reynolds numbers and small roughness: see figure 2.4(a). The modified $F_{\text {turb }}$ fixes this quite effectively. Figure 2.4(b) shows a reasonable laminar region over the front part of the suction surface.

The constants in equation (2.9) were calibrated with the correlation-based model in Boyle and Stripf (2009) for a high pressure turbine blade case which was experimentally tested by Stripf et al. (2005) and Stripf (2007). The quantity $F_{Q}^{1.5} / 350$ is plotted in figure 2.5. It is nonzero only in a very thin region of strong acceleration, on the suction surface. More details about this test case are introduced in the next section.

For high roughness, the modified $R_{\nu}$ may exceed the upper bound of 100, in $G_{\gamma}$, even close to the wall. This issue can be solved by increasing this upper bound as follows

$$
\begin{aligned}
G_{\gamma}= & 7.5 \max \left[0, \min \left(\left(100+R_{c 3}\right)-R_{\nu}, 1\right)\right] \\
& \times \min \left[\max \left(R_{\nu}-\left(18+R_{c 2}\right), 0\right), 1\right] . \\
R_{c 3} & =0.3 \frac{\left(d+C_{r} r\right)^{2} \sqrt{|Q|} \operatorname{sign}(Q)}{\nu}, \\
R_{c 3} & =\max \left[\min \left(R_{c 3}, 100\right),-100\right] .
\end{aligned}
$$

$R_{c 3}$ is again a function of $Q$ so that it becomes active in the acceleration region. It uses the effective wall distance instead of the roughness height $r$; the aim is to take into account pressure gradient effects, even for smooth wall cases. Favorable pressure gradients can 


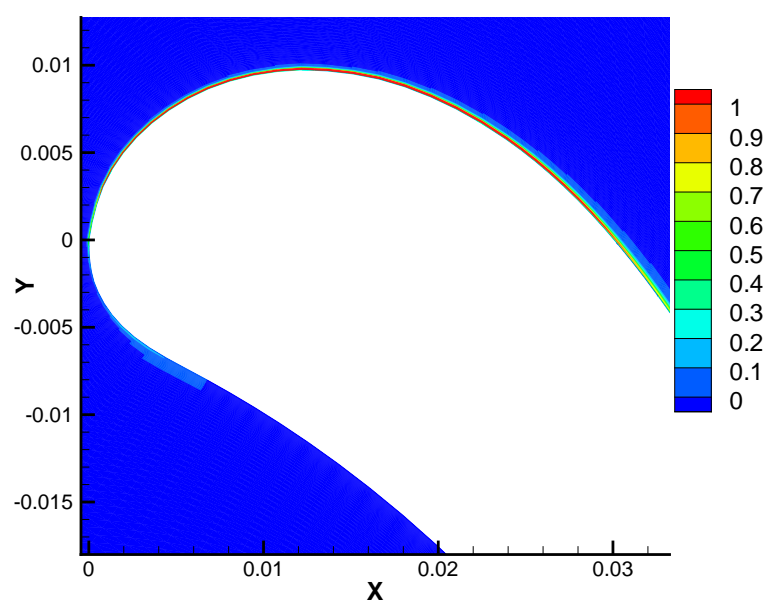

Figure 2.5: Contours of quantity $F_{Q}^{1.5} / 350$. This test case is the same as figure 2.4.

enhance the sink term and delay the transition. Adverse pressure gradients can suppress the sink term and accelerate the transition. The limiters in equation (2.12) make the modified upper bound of $R_{\nu}$ range between 0 and 200 .

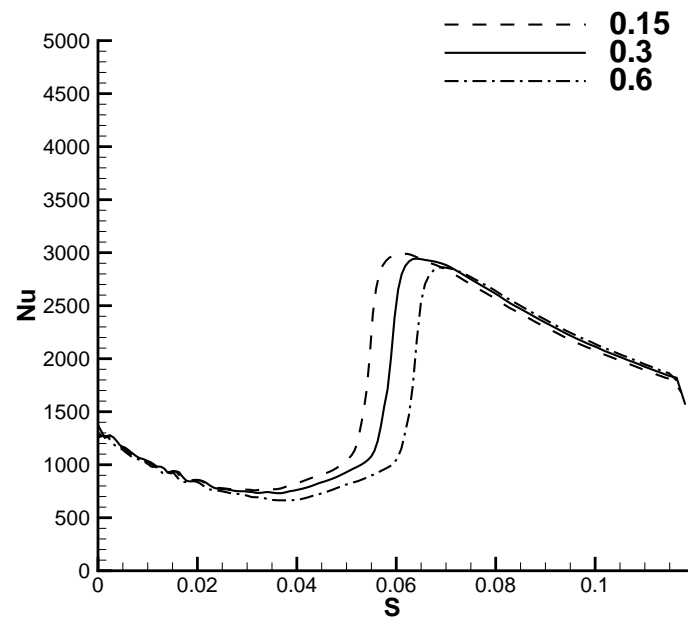

Figure 2.6: Effects of the multiplicative coefficient in $R_{c 3}$. This test case uses the Stripf's turbine, with inflow chord Reynolds number $R e_{c}=2.5 \times 10^{5}$, turbulence intensity at the mid-pitch of the leading edge location $T u_{l . e .}=1.6 \%$, and roughness height $r=0.73 \times 10^{-4} \mathrm{~m}$.

The coefficient 0.3 in $R_{c 3}$ is calibrated on Stripf's turbine test cases. Its effect on $C_{f}$ is depicted in figure 2.6. This shows the Nusselt number distribution over the suction surface versus the surface coordinate. When this coefficient is halved the transition is accelerated. When it is doubled, the opposite effect is observed. The selected value, 0.3, gives good 
agreements with Stripf's data for a wide range of roughness heights, Reynolds numbers and free-stream turbulence intensities.

\section{Computation and results}

\subsection{Flat plate cases}

The set up for this case has been described in section 2.1. It is modified from the T3A test case (Roach and Brierley, 1992). The only change of the inflow condition is the turbulence intensity, $T u_{i n}$. Two values, $T u_{i n}=1.5 \%$ and $3.5 \%$, are specified. Another difference is the wall boundary conditions for $k$ and $\omega$ : equations (1.6) and (1.4) are used. Grid independence has been checked. The first grid point was refined down to $y_{1}^{+} \approx 0.1$, to show grid independence in the flat plate, rough wall cases. $y_{1}^{+}$was 1.0 in the smooth wall cases in Ge et al. (2014). This is consistent with the grid requirements for Knopp's model: from grid independence tests, $y_{1}^{+} \lesssim 0.3$ is sufficient for accurate velocity profiles in the fully turbulent region over rough surfaces.

A prerequisite of the current modeling is that the transition location computed by the smooth wall transition model should not shift when only the roughness boundary conditions for $k$ and $\omega$ are imposed, because the roughness should not affect the laminar boundary layer (at least, for relative low roughness heights). The only influence should be an increase of the $C_{f}$ values in the fully turbulent region, compared to the smooth wall case.

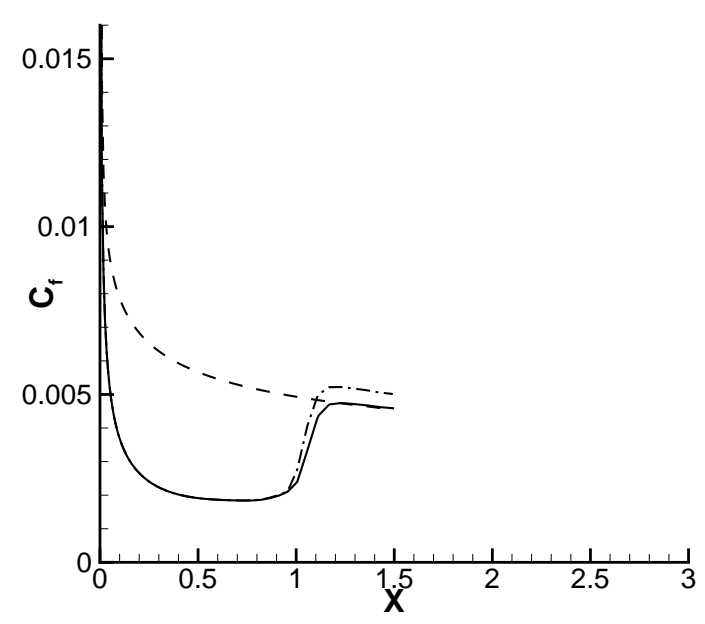

Figure 3.1: Contribution of the smooth Leading edge. The smooth wall case (solid), the case with the rough wall boundary conditions and uniform roughness (dash), and the case with the rough wall boundary conditions and a smooth leading edge (dash-dot)

However, this prerequisite cannot be satisfied unless a tiny, smooth leading edge region is specified. The reason is obvious: for an ideal, flat plate, the sharp leading edge leads to infinite skin friction coefficient $C_{f} \sim 1 / \sqrt{x}$ as $x \rightarrow 0$; therefore, $r^{+}$becomes infinite, which is physically unacceptable. If the roughness boundary conditions are imposed at the leading 


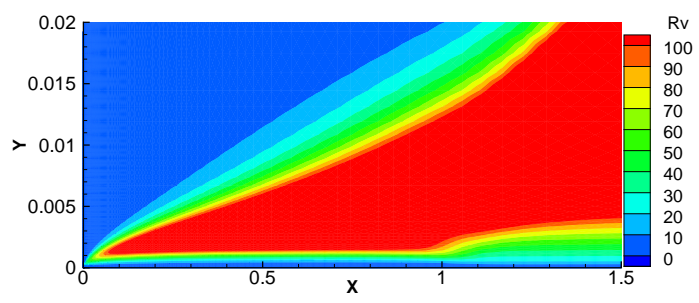

(a) $R_{\nu}$ with a smooth wall.

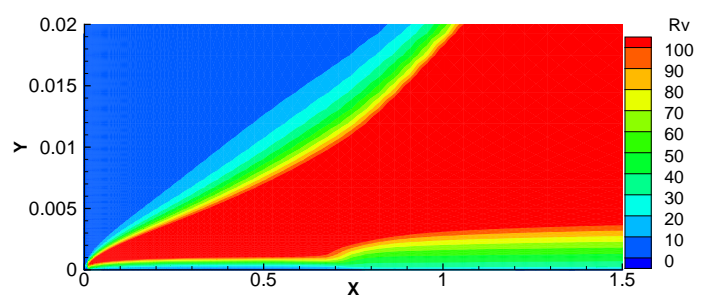

(c) $R_{\nu}$ with $r=10 \times 10^{-4} \mathrm{~m}$.

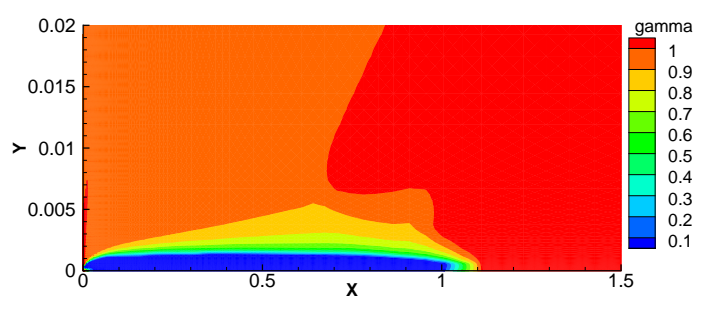

(b) $\gamma$ with a smooth wall.

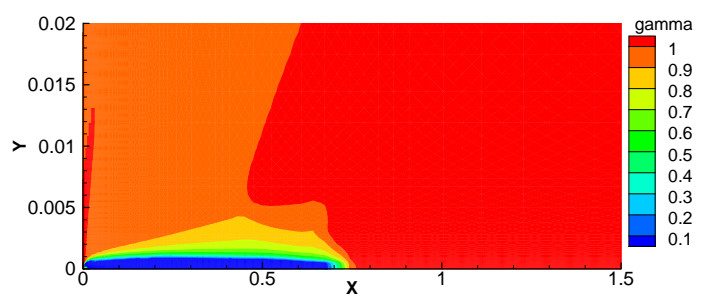

(d) $\gamma$ with $r=10 \times 10^{-4} m$.

Figure 3.2: Near wall distributions of $R_{\nu}$ and $\gamma$ compared between smooth and rough walls

edge, the very large $r_{+}$causes the boundary layer to transition immediately. Numerically, the computation converges to a fully turbulent solution. For a realistic geometry, with a rounded leading edge, with a stagnation point, $C_{f}$ would be finite.

The need for a smooth leading edge is illustrated in figure 3.1 where $T u_{i n}=1.5 \%$ and $r=7 \times 10^{-4} m\left(r^{+} \approx 12\right)$. The solid line represents the solution for the smooth wall case. When boundary conditions (1.6) and (1.4) for $k$ and $\omega$ are imposed on a wall with uniform roughness, the result is the dash line. When a tiny smooth region $(r=0 m$ if $x<0.01 m)$ is imposed, the $C_{f}$ curve is the dash-dot line. The transition location predicted in this case is the same as the smooth wall case, with greater $C_{f}$ values in the fully turbulent region.

With this caveat about the leading edge, the new roughness modification is validated. Contours of $R_{\nu}$ and $\gamma$, with and without roughness, are plotted in figure 3.2. Contours of $R_{\nu}$ with a rough wall in figure 3.2(c) show higher values near the wall than those in figure 3.2 (a) with a smooth wall, as if there is a displacement of the wall origin upward into the flow field in going from the smooth wall case to the rough wall case. The contours of $\gamma$ in figure 3.2(b) and 3.2(d) depict the laminar region moving upstream, as the roughness increases.

The $C_{f}$ curves predicted by the current model for several different roughness heights are displayed in figure 3.3. As the roughness increases, the transition location moves upstream toward the leading edge. In the fully turbulent region, the $C_{f}$ values become larger with higher roughness. For higher turbulence intensity, figure 3.3(b) shows that the transition 


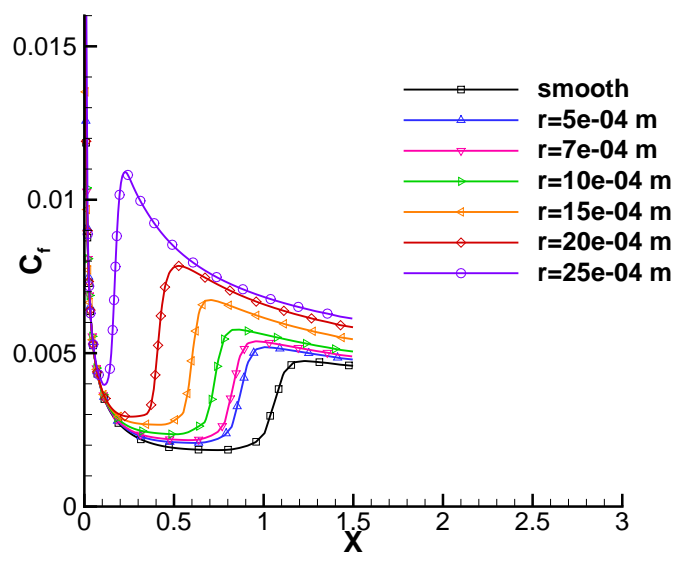

(a) $T u_{\text {in }}=1.5 \%$.

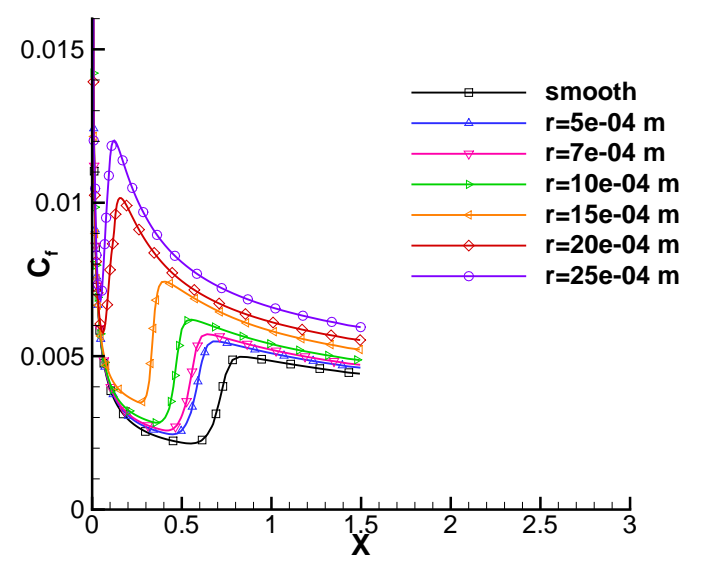

(b) $T u_{\text {in }}=3.5 \%$.

Figure 3.3: Skin friction for flat plate cases with different roughness heights and different inflow turbulence intensities.

locations become less sensitive to the roughness height. Similarly, for higher roughness, the transition locations become less sensitive to the free-stream turbulence intensity.

Reasonable agreement is observed between the critical Reynolds number $R e_{\theta t}$ predicted by the model and that calculated from the correlation equation (1.7). Table 3.1 contains details for the case with $T u_{i n}=1.5 \%$. For the majority of the roughness heights, the predicted $R e_{\theta t}$ is a little overestimated. For large roughness heights, when $R e_{\theta t}<200$, the model tends to predict early transition.

Note that, from the $C_{f}$ curves, it is very ambiguous which point, exactly, is the transition location. The same question arises for the experimental data. In the present case, the point where the $C_{f}$ curves start to rise is assumed to be the onset of transition. To calculate the momentum thickness at transition onset, $\theta_{t}$, the velocity profile is extracted and integrated. This quantity along with local free-stream $T u$ and $C_{f}$ may introduce errors in evaluating both the predicted and correlated $R e_{\theta t}$. In addition, a wide range of critical Reynolds numbers are obtained from cases with various roughness heights, whereas the number of parameters in the present model is relatively few. Thus, very precise prediction of $R e_{\theta t}$ is not expected.

\subsection{High pressure turbine blade}

In this section, 2-D flow through a passage of a high pressure turbine (HPT) blade cascade is tested with variation of inlet turbulence intensity, Reynold number and roughness height. An experimental database is available in Stripf et al. (2005) and Stripf (2007).

Coordinates for the turbine blade geometry are provided by Stripf (2007). The true chord is $93.95 \mathrm{~mm}$ and the axial chord is about $53 \mathrm{~mm}$. The blade pitch is $81.26 \mathrm{~mm}$. Figure 
Table 3.1: Summary of roughness heights and transition locations (T3A: $T u_{i n}=1.5 \%$ ).

\begin{tabular}{lcccccc}
\hline$r\left(\times 10^{-4} m\right)$ & 5 & 7 & 10 & 15 & 20 & 25 \\
\hline$r^{+}$ & 8.5 & 12.0 & 17.4 & 27.2 & 37.5 & 48.0 \\
\hline$X_{t}$ & 0.83 & 0.78 & 0.67 & 0.48 & 0.35 & 0.11 \\
\hline$R e_{\theta t-p r}$ & 412 & 401 & 372 & 303 & 255 & 132 \\
\hline$R e_{\theta t-c r}$ & 427 & 394 & 346 & 282 & 226 & 173 \\
\hline$R e_{\theta t-\text { smooth }}$ & & & 442 & & & \\
\hline
\end{tabular}

The subscript "pr" means predicted, and "cr" means correlated.

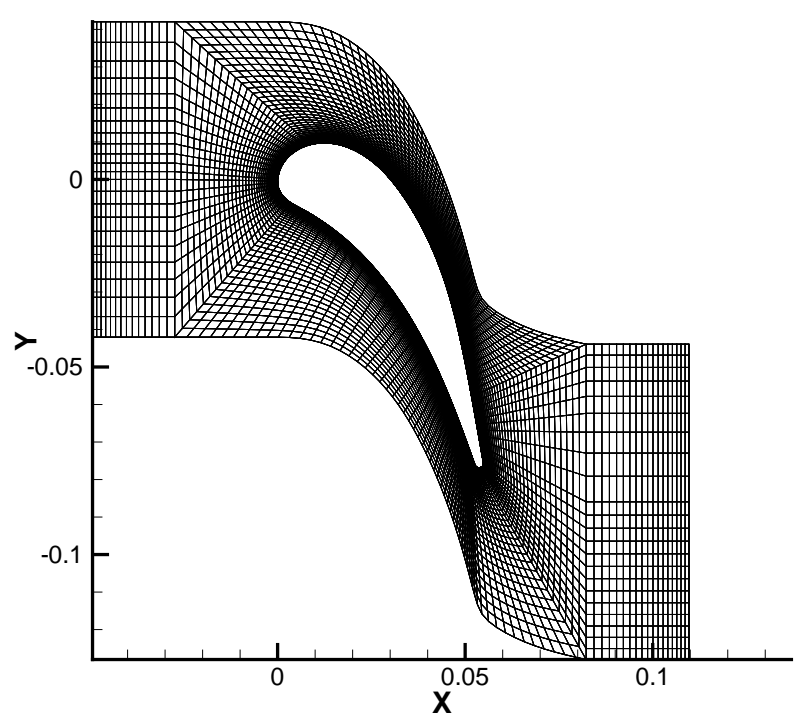

Figure 3.4: Mesh used to the HPT blade case showing every other line in tangential and normal wall directions.

3.4 displays the mesh for this case. It is a 3-block grid with an O-grid block as the center part around the blade and two H-grid blocks as the extended inlet and outlet passages. The flow is incompressible.

Cyclic boundary conditions are imposed on the top and bottom boundaries. The reference pressure is set zero at the outlet and the pressure gradient is zero at the inlet. Table 3.2 lists the flow conditions for all test cases. The inlet velocity has only the $x$-component with the value shown as $U_{i n}$. $T u_{l . e \text {. }}$ and $R_{t-\text { l.e. }}$ represent the turbulence intensity and Reynolds number $(k / \omega \nu)$ at the mid-pitch of the leading edge location. The kinematic viscosity is $1.5 \times 10^{-5} \mathrm{~m}^{2} \mathrm{~s}^{-1}$. The inflow Reynolds number based on the true chord $R e_{c}$ is $2.5 \times 10^{5}$ for TC1-2, and $1.4 \times 10^{5}$ for TC3-4. The number of grid points of the O-grid block is $321 \times 101$ for the high $R e_{c}$ and $161 \times 101$ for the low $R e_{c}$, in circumferential and wall normal directions respectively. The $\mathrm{H}$-grid blocks have $15 \times 46$ and $19 \times 46$ grid points in $x$ and $y$ directions at the inlet and outlet for the high $R e_{c}$, and $15 \times 23$ and $19 \times 23$ for the low $R e_{c}$. The first 
grid spacing is set to $y_{1}^{+} \approx 0.5$ for both grids.

To compute the heat transfer to the blade surface, the energy equation is solved along with the continuity and momentum equations. An effective thermal diffusivity $\kappa_{\text {eff }}=$ $\nu / \operatorname{Pr}+\nu_{T} / P r_{T}$ is used to represent heat transport. The laminar and turbulent Prandtl numbers, $\operatorname{Pr}$ and $\operatorname{Pr}_{T}$, are 0.72 and 0.86 , respectively. The air density is $1.2 \mathrm{~kg} / \mathrm{m}^{3}$ and the heat capacity $c_{p}$ is $1000 \mathrm{~m}^{2} /\left(s^{2} K\right)$. A constant surface temperature, $300^{\circ} K$, is specified, and the ambient temperature is $400^{\circ} \mathrm{K}$.

\begin{tabular}{ccccc} 
Table 3.2: & Summary of inlet conditions for the Stripf's HPT blade case \\
\hline Case & $U_{\text {in }}(m / s)$ & $T u_{l . e .}(\%)$ & $R_{t-l . e .}$ & $\nu\left(\times 10^{-5} m^{2} s^{-1}\right)$ \\
\hline TC1 & 39.915 & 2.4 & 12.0 & 1.5 \\
TC2 & 39.915 & 1.6 & 5.5 & 1.5 \\
TC3 & 22.352 & 4.3 & 21.6 & 1.5 \\
TC4 & 22.352 & 2.7 & 8.4 & 1.5
\end{tabular}

The computed results are plotted in figure 3.5. The Nusselt numbers predicted by the current model on the suction surface are compared with the predicted heat transfer coefficient by Boyle's correlation-based model. Reasonable agreement is achieved, in term of the shape of the curves, and more importantly the transition location. As the roughness height increases, the transition location moves upstream, to the leading edge.

Comparison between figures 3.5(a) and (b) shows that for relative high roughness, the transition onset becomes less sensitive to the free-stream $T u$. This observation agrees with the experimental database. Similarly, high $T u$ leads to less sensitivity to the roughness height. In addition, one sees that the transition location on the rough wall is more sensitive to the chord Reynolds number than that on the smooth wall. This is shown by comparing $3.5(\mathrm{a})$ and $(\mathrm{c})$, or $3.5(\mathrm{~b})$ and $(\mathrm{d})$.

There is some uncertainty about the free-stream turbulence in these experiments. The inflow $k$ and $\omega$ were adjusted for the current simulations so that the solid and dashed, red curves provide similar transition locations in the smooth wall case. The experimental data suggest a much quicker decay than the $k-\omega$ model does, even if a very high dissipation rate, $\omega$, is specified to the free-stream. Therefore, our method to determine the inlet $k$ and $\omega$ is to make the transition location the same as the experimental data for the smooth wall case, then use this in the roughness computations.

In the fully turbulent region, it is reasonable that the heat transfer rate becomes higher as the roughness increases. However in the experimental data, heat transfer is not very sensitive to the roughness height. The dimensionless roughness $r^{+}$is evaluated from $C_{f}$ close to the trailing edge and listed in tables 3.3 and 3.4. Only the highest roughness reaches the fully rough condition $\left(r^{+}>90\right)$. Most of the cases are transitionally rough, which should cause sensitivity to the roughness geometry in the fully turbulent region. In the experiment, the boundary layer may have not developed into a fully turbulent condition, even after transition. 


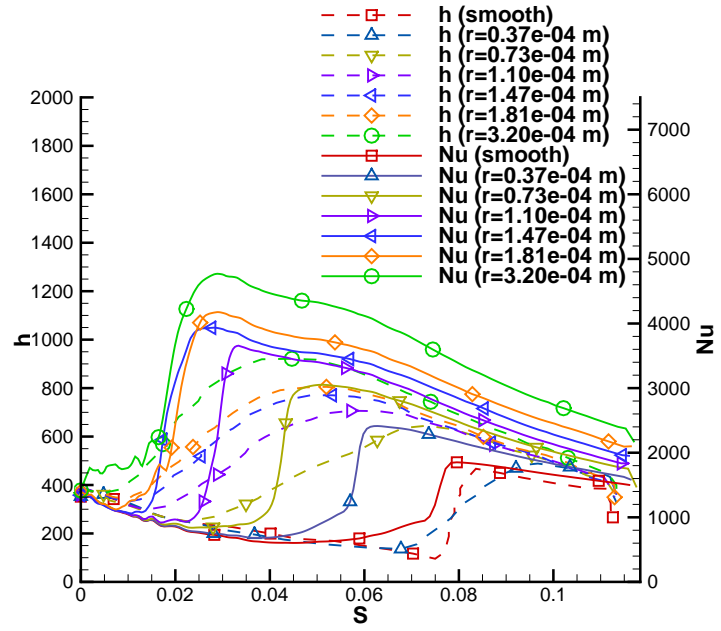

(a) TC1.

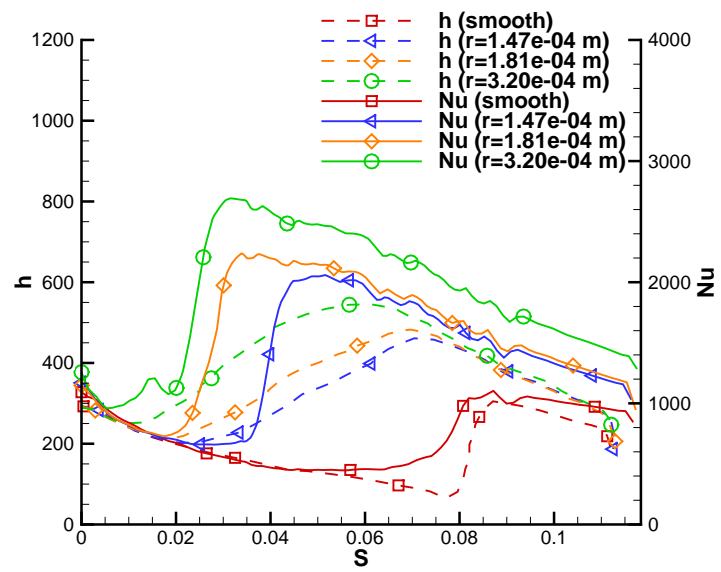

(c) TC3.

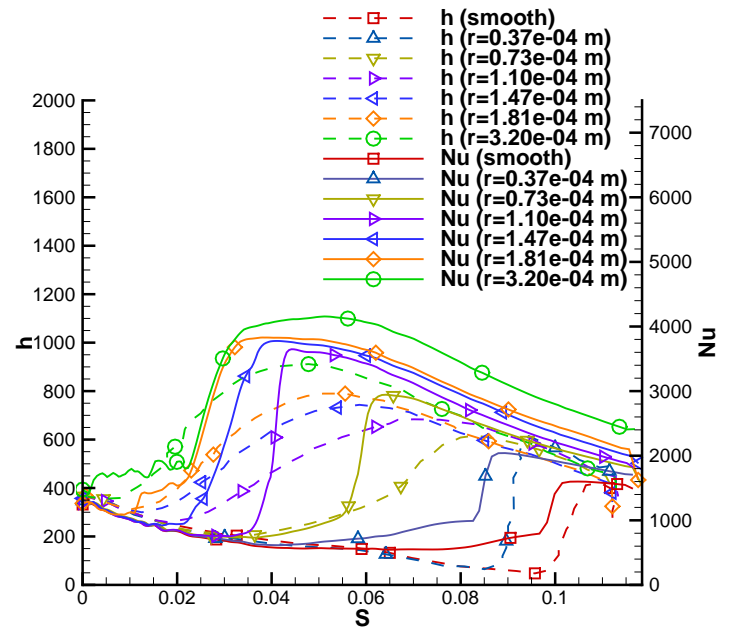

(b) TC2.

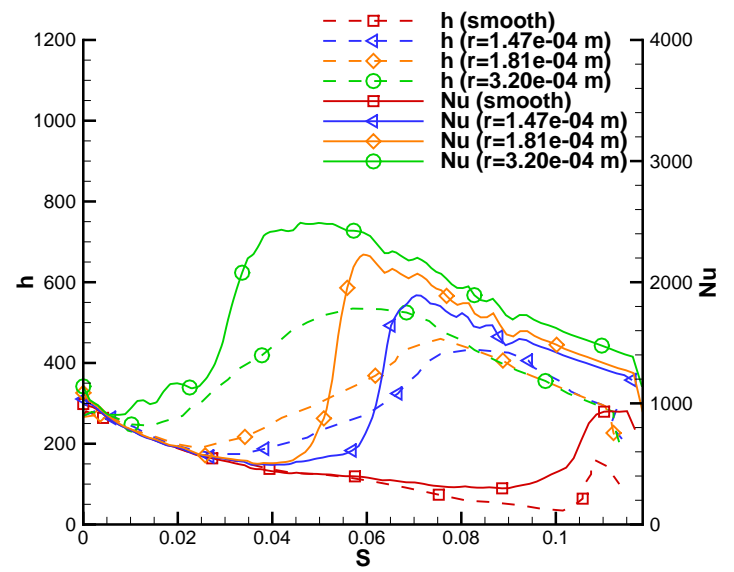

(d) TC4.

Figure 3.5: Results for the Stripf's HPT blade case. The solid lines are the predicted curves for the Nusselt number by the current model, and the dash lines are the predicted curves for the heat transfer coefficient by the correlation-based model in Boyle and Stripf (2009). 
Table 3.3: Summary of roughness heights for the Stripf's HPT blade, TC2

\begin{tabular}{lcccccc}
\hline$r\left(\times 10^{-4} m\right)$ & 0.37 & 0.73 & 1.10 & 1.47 & 1.81 & 3.20 \\
\hline$r^{+}$ & 16.1 & 32.0 & 47.7 & 65.3 & 82.0 & 153.3 \\
\hline
\end{tabular}

Table 3.4: Summary of roughness heights for the Stripf's HPT blade, TC4

\begin{tabular}{llll}
\hline$r\left(\times 10^{-4} m\right)$ & 1.47 & 1.81 & 3.20 \\
\hline$r^{+}$ & 40.0 & 49.3 & 90.0 \\
\hline
\end{tabular}

\subsection{Low pressure turbine blade}

The database provided by Stripf (2007) also contains a low pressure turbine (LPT) blade cascade. This may serve as a good supplementary validation of the roughness model. Stripf et al. (2009b) and Boyle and Stripf (2009) use this case to validate their correlationbased model. In addition to the effect of roughness height, some other factors, such as roughness density, and locally varying roughness are investigated for this case in their papers. Since the current model considers only the effective sand grain roughness height $r$, the influence of various $r$ evaluated by Boyle and Stripf (2009) for different rough surfaces are examined in this section along with two different free-stream $\mathrm{Tu}$. The predicted results are compared with the measured data given in Stripf et al. (2009b).

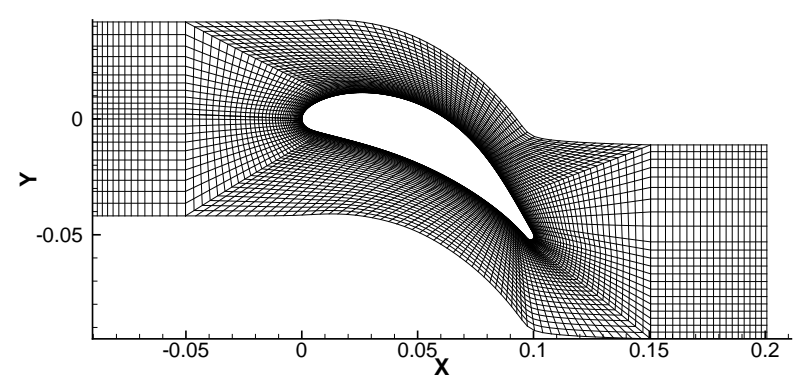

Figure 3.6: Mesh used to the LPT blade case showing every other line in tangential and normal wall directions.

The geometry is again obtained from Stripf (2007). The true chord is $113.34 \mathrm{~mm}$ and the axial chord is about $100 \mathrm{~mm}$. The blade pitch is $83.4 \mathrm{~mm}$. The same approach as the HPT case is used to generate the LPT mesh; see figure 3.6. The size of the O-grid block is $321 \times 101$ with $y_{1}^{+} \approx 0.5$. The H-grid blocks have $15 \times 46$ and $19 \times 46$ grid points at the inlet and outlet. Similar boundary conditions are employed as the HPT case. The flow parameters are shown in table 3.5. The true chord Reynolds number $R e_{c}$ is $2.5 \times 10^{5}$. Parameters for the energy equation are all the same as the previous case.

The computed results are depicted in figure 3.7. Decent agreement is observed between the Nusselt number distribution predicted by the current model and measured in the experiment on the suction surface. The effect of the roughness height is shown clearly. The 
Table 3.5: Summary of inlet conditions for the Stripf's LPT blade case.

\begin{tabular}{lcccc}
\hline Case & $U_{i n}(\mathrm{~m} / \mathrm{s})$ & $T u_{\text {l.e. }}(\%)$ & $R_{t-l . e .}$ & $\nu\left(\times 10^{-5} \mathrm{~m}^{2} \mathrm{~s}^{-1}\right)$ \\
\hline TC5 & 33.086 & 3.1 & 30.0 & 1.5 \\
TC6 & 33.086 & 2.7 & 22.3 & 1.5
\end{tabular}

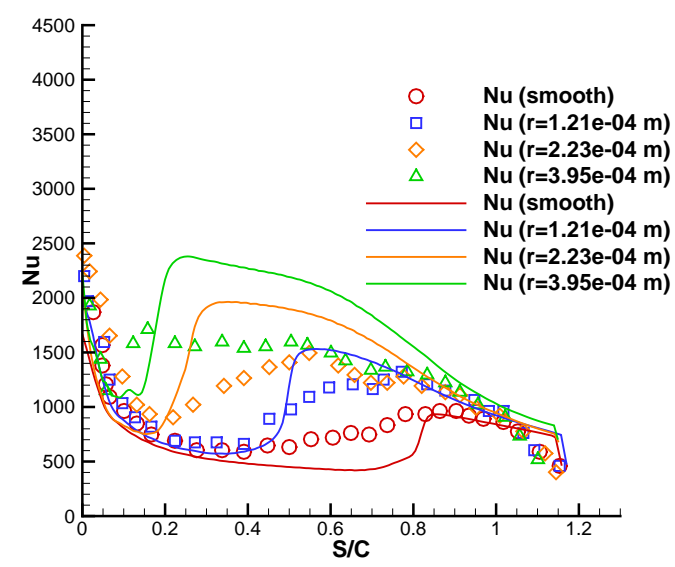

(a) TC5.

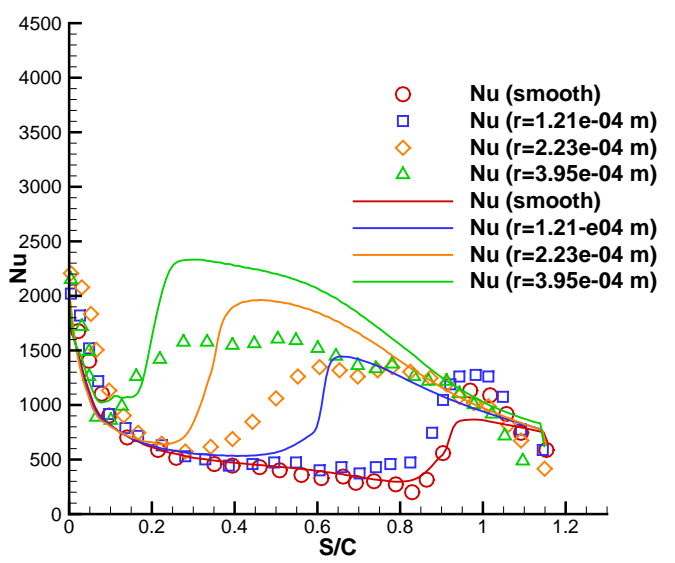

(b) TC6.

Figure 3.7: Results for the Stripf's LPT blade case. The solid lines are the predicted curves for the Nusselt number by the current model, and the symbols present the experimental data.

Table 3.6: Summary of roughness heights for the Stripf's LPT blade, TC6

\begin{tabular}{llll}
\hline$r\left(\times 10^{-4} m\right)$ & 1.21 & 2.23 & 3.95 \\
\hline$r^{+}$ & 14.0 & 22.7 & 36.2 \\
\hline
\end{tabular}

inflow conditions for $k$ and $\omega$ for this case are again chosen based on the criterion that computed results of the smooth wall cases should match the experimental data. By comparing the results with two different $T u$, one can infer that the transition location becomes less sensitive to $T u$ as the roughness becomes higher, which is both presented by the experimental data and by the current model. These observations are consistent with the HPT blade case. Various $r^{+}$for TC6 are given in table 3.6, which are smaller than those in the HPT case with comparable values of $r$, due to lower skin friction.

\section{Conclusions}

Based on the concept of the effective displacement of the origin, an intermittency model for bypass transition was extended to predict roughness effects on transition. The local Reynolds number was modified by adding an effective displacement to the wall distance. Some other modifications are needed for practical cases, like a turbine blade cascade. 
Boundary conditions for the fully turbulent region of the boundary layer on rough walls were imposed. The model was validated by flat plate and turbine blade cases, with variations of roughness height, Reynolds number, free-stream turbulence intensity and pressure gradient. Reasonable agreement, especially in terms of transition location, with the results of a data correlation-based model, or with experimental data, was obtained.

The performance of the model for predicting roughness effects depends on the performance of the model for smooth wall cases. The key point is that the roughness modification is capable of properly shifting the smooth wall results for boundary layer transition. Roughness effects on transition in separated flow are not in the scope of the current work; they remain to be explored in the future.

\section{Acknowledgments}

This work was funded by NSF grant \#CBET-1228195.

\section{Appendix: full model formulation}

\section{A.1. Model for smooth walls}

The intermittency transport equation is as follows,

$$
\begin{gathered}
\frac{D \gamma}{D t}=\partial_{j}\left[\left(\frac{\nu}{\sigma_{l}}+\frac{\nu_{T}}{\sigma_{\gamma}}\right) \partial_{j} \gamma\right]+P_{\gamma}-E_{\gamma}, \\
P_{\gamma}=F_{\gamma}|\Omega|\left(\gamma_{\max }-\gamma\right) \sqrt{\gamma} \\
E_{\gamma}=G_{\gamma} F_{\text {turb }}|\Omega| \gamma^{1.5},
\end{gathered}
$$

where $\sigma_{l}=5.0, \sigma_{\gamma}=0.2$ and $\gamma_{\max }=1.1$. The model depends on the parameters

$$
\left\{\begin{array}{l}
R_{t} \equiv \frac{\nu_{T}}{\nu}, \\
T_{\omega} \equiv R_{t} \frac{|\Omega|}{\omega}, \\
R_{\nu} \equiv \frac{d^{2}|\Omega|}{2.188 \nu} .
\end{array}\right.
$$

Here $|\Omega|$ is defined by $\sqrt{2 \Omega_{i j} \Omega_{i j}}$. The source term contains

$$
\begin{aligned}
F_{\gamma}=2 & \max \left[0, \min \left(100-0.7 R_{\nu}, 1\right)\right] \\
& \times \min \left[\max \left(R_{\nu}-R_{c 1}, 0\right), 4\right] . \\
R_{c 1} & =400-360 \min \left(\frac{T_{\omega}}{2}, 1\right) .
\end{aligned}
$$


The sink term contains

$$
\begin{gathered}
G_{\gamma}=7.5 \max \left[0, \min \left(100-R_{\nu}, 1\right)\right] \\
\times \min \left[\max \left(R_{\nu}-18,0\right), 1\right] . \\
F_{\text {turb }}=e^{-\left(R_{\nu} R_{t}\right)^{1.2}} .
\end{gathered}
$$

The separation modification is applied via $\gamma_{e f f}$. It depends on adverse pressure gradient detector,

$$
R_{s} \equiv d \cdot \frac{\mathbf{n}_{w} \cdot \nabla|S| \omega}{\sqrt{2}|S|^{2}} .
$$

Here $|S|$ is defined by $\sqrt{S_{i j} S_{j i}}$.

$$
\gamma_{\text {eff }}=\max \left[\min (1, \gamma), \min \left(2, F_{R_{t}} F_{R_{\nu}} F_{R_{s}}\right)\right] .
$$

The limiter $\min (1, \gamma)$ clips off values greater than one for the $\gamma$ field solved by the $\gamma$ transport equation. The 3 functions are defined as follows,

$$
\begin{gathered}
F_{R_{t}}=e^{-\left(R_{t} / 10\right)^{3}}, \\
F_{R_{\nu}}=\max \left(R_{\nu}-200,0\right), \\
F_{R_{s}}=\min \left[1.0, \max \left(10+5 R_{s}, 0\right)\right] \\
\times \min \left[1.0, \max \left(10-5 R_{s}, 0\right)\right] .
\end{gathered}
$$

The current formulation is applied to the $k-\omega$ RANS closure. The production term of the $k$ equation is multiplied by $\gamma_{e f f}$. This is the only appearance of $\gamma$ within the turbulence model.

$$
\begin{gathered}
\frac{D k}{D t}=P_{k}-C_{\mu} k \omega+\partial_{j}\left[\left(\nu+\frac{\nu_{T}}{\sigma_{k}}\right) \partial_{j} k\right], \\
P_{k}=\min \left(2 \nu_{T}|S|^{2}, k|S| / \sqrt{3}\right) \gamma_{e f f}, \\
\frac{D \omega}{D t}=2 C_{\omega 1}|S|^{2}-C_{\omega 2} \omega^{2}+\partial_{j}\left[\left(\nu+\frac{\nu_{T}}{\sigma_{\omega}}\right) \partial_{j} \omega\right],
\end{gathered}
$$

where $C_{\mu}=0.09, C_{\omega 1}=5 / 9, C_{\omega 2}=3 / 40$ and $\sigma_{\omega}=\sigma_{k}=2$. The eddy viscosity $\nu_{T}$ is $k / \omega$. 


\section{A.2. Model for rough walls}

An effective origin for the wall distance is applied to the model for smooth walls.

$$
\begin{gathered}
R_{\nu} \equiv \frac{(d+0.26 r)^{2}|\Omega|}{2.188 \nu} . \\
R_{s} \equiv(d+0.26 r) \cdot \frac{\mathbf{n}_{w} \cdot \nabla|S| \omega}{\sqrt{2}|S|^{2}} .
\end{gathered}
$$

Accordingly, the sink term has to be modified due to the change of $R_{\nu}$.

$$
\begin{gathered}
G_{\gamma}=7.5 \max \left[0, \min \left(\left(100+R_{c 3}\right)-R_{\nu}, 1\right)\right] \\
\times \min \left[\max \left(R_{\nu}-\left(18+R_{c 2}\right), 0\right), 1\right] . \\
R_{c 2}=3.0\left[\frac{(0.26 r)^{2}|\Omega|}{2.188 \nu}\right]^{0.8} \cdot \\
R_{c 3}=0.3 \frac{(d+0.26 r)^{2} \sqrt{|Q|} \operatorname{sign}(Q)}{\nu}, R_{c 3}=\max \left[\min \left(R_{c 3}, 100\right),-100\right],
\end{gathered}
$$

where $Q=\Omega_{i j} \Omega_{i j}-S_{i j} S_{i j}$.

$$
\begin{gathered}
F_{\text {turb }}=e^{-\left(R_{\nu_{\text {new }}} R_{t}\right)^{1.2}} . \\
R_{\nu_{\text {new }}}=R_{\nu} e^{-F_{Q}^{1.5} / 350} . \\
F_{Q}=\max \left[0, \frac{r^{2} \sqrt{|Q|} \operatorname{sign}(Q)}{\nu}\right] .
\end{gathered}
$$

$R_{c 3}$ and $R_{\nu_{\text {new }}}$ are used for strong favorable pressure gradient cases, such as a region right after the leading edge on a suction surface of a turbine blade.

\section{References}

Arts, T., Lamvert de Rouvroit, M., Rutherford, A., 1990. Aero-thermal investigation of a highly loaded transonic linear turbine guide vane cascade. VKI Technical Note 174.

Boyle, R., Senyitko, R., 2003. Measurements and predictions of surface roughness effects on turbine vane aerodynamics. ASME Paper GT-2003-38580.

Boyle, R., Stripf, M., 2009. Simplified approach to predicting rough surface transition. Journal of Turbomachinery 131, 041020.

Dassler, P., Kožulović, D., Fiala, A., 2010. Modelling of roughness-induced transition using local variables, in: V European Conference on CFD, ECCOMAS CFD. 
Durbin, P., 2012. An intermittency model for bypass transition. International Journal of Heat and Fluid Flow 36, 1-6.

Durbin, P., Medic, G., Seo, J.M., Eaton, J., Song, S., 2001. Rough wall modification of two-layer $k-\varepsilon$. Journal of Fluids Engineering 123, 16-21.

Durbin, P.A., 2009. Limiters and wall treatments in applied turbulence modeling. Fluid Dynamics Research 41, 012203 (18pp).

Elsner, W., Warzecha, P., 2014. Numerical study of transitional rough wall boundary layer. Journal of Turbomachinery 136, 011010.

Ge, X., Arolla, S., Durbin, P., 2014. A bypass transition model based on the intermittency function. Flow, Turbulence and Combustion 93, 37-61.

Knopp, T., Eisfeld, B., Calvo, J.B., 2009. A new extension for k turbulence models to account for wall roughness. International Journal of Heat and Fluid Flow 30, $54-65$.

Langtry, R., Menter, F., 2009. Correlation-based transition modelling for unstructured parallelized computational fluid dynamics codes. AIAA Journal 47, 2894-2906.

Mayle, R.E., 1991. The 1991 igti scholar lecture: The role of laminar-turbulent transition in gas turbine engines. Journal of Turbomachinery 113, 509-536.

Menter, F., Langtry, R., Völker, S., 2006. Transition modelling for general purpose CFD codes. Flow, Turbulence and Combustion 77, 277-303.

Roach, P.E., Brierley, D.H., 1992. The influence of a turbulent free-stream on zero pressure gradient transitional boundary layer development Part 1: Test cases T3A and T3B, in: Numerical Simulation of Unsteady Flows and Transition to Turbulence, ERCOFTAC. pp. 319-347.

Seo, J.M., 2004. Closure Modeling and Numerical Simulation for Turbulent Flows: Wall Roughness Model, Realizability, and Turbine Blade Heat Transfer. Ph.D. thesis. Flow Physics and Computation Division, Dept. of Mechanical Engineering, Stanford University.

Stripf, M., 2007. Einfluss der oberflächenrauigkeit auf die transitionale grenzschicht an gasturbinenschaufeln. Forschungsberichte aus dem Institut für Thermische Strömungsmaschinen 38.

Stripf, M., Schulz, A., Bauer, H.J., Wittig, S., 2009a. Extended models for transitional rough wall boundary layers with heat transfer part I: Model formulations. Journal of Turbomachinery 131, 031016.

Stripf, M., Schulz, A., Bauer, H.J., Wittig, S., 2009b. Extended models for transitional rough wall boundary layers with heat transfer part II: Model validation and benchmarking. Journal of Turbomachinery 131, 031017. 
Stripf, M., Schulz, A., Wittig, S., 2005. Surface roughness effects on external heat transfer of a hp turbine vane. Journal of Turbomachinery 127, 200-208.

Wilcox, J., 1998. Turbulence modeling for CFD (Second Edition). DWC Industries, La Canada. 